\title{
Prenatal stress and anxiety is associated with lower dietary fat and dairy consumption among healthy pregnant women
}

\author{
K. Lindsay, C. Buss, P. Wadhwa and S. Entringer \\ UCI Development Health and Disease Research Program, University of California Irvine, School of Medicine, Irvine, \\ CA 92697, USA
}

Two of the most independently studied prenatal factors to influence fetal development and pregnancy/offspring outcome are maternal nutrition and psychosocial stress. Yet few studies have concurrently examined diet-stress interactions in pregnancy. Evidence from non-pregnant animal and human studies suggests that physiological and hormonal response to psychosocial stress induces increased consumption of palatable foods high in fat and sugar. ${ }^{(1)}$ There are some reports from cross-sectional studies in pregnancy that lower maternal fish consumption increases the risk of depressive symptoms and anxiety ${ }^{(2,3)}$, which has been attributed to low intake of omega-3 fatty acids. The aim of this study is to prospectively assess maternal dietary intakes, stress and anxiety across gestation to investigate if psychosocial state is associated with diet quality, macronutrient distribution or intake of specific food groups.

Non-diabetic pregnant women $(\mathrm{N}=173)$ were recruited in early pregnancy and followed prospectively with research laboratory visits in each trimester. At each visit, women completed validated questionnaires to evaluate perceived stress and trait anxiety and dietary intakes were assessed using the $24-\mathrm{hr}$ recall method. Two further $24-\mathrm{hr}$ recalls were conducted via telephone such that intakes were assessed over three non-consecutive days in each trimester, including one weekend day. Average pregnancy values were computed for stress and anxiety scores and the following dietary intakes: macronutrients as a percent of total energy ( \%TE), glycaemic index (GI), food group servings, and the Alternative Healthy Eating Index adapted for pregnancy (AHEI-P); a validated measure of overall dietary quality ${ }^{(4)}$. All variables had a normal distribution except for food group servings which were log-transformed.

Table 1. Pearson correlations of mean pregnancy stress and anxiety scores with diet variables

\begin{tabular}{|c|c|c|c|c|}
\hline \multirow[t]{2}{*}{ Mean dietary intake } & \multicolumn{2}{|c|}{ Mean trait anxiety score } & \multicolumn{2}{|c|}{ Mean perceived stress score } \\
\hline & $\bar{r}$ & $\overline{p \text {-value }}$ & $\overline{\mathrm{r}}$ & $\overline{p \text {-value }}$ \\
\hline$\%$ TE total fat & -0.191 & $0 \cdot 015$ & $-0 \cdot 191$ & 0.011 \\
\hline$\%$ TE saturated fat & & & -0.167 & $0 \cdot 025$ \\
\hline$\%$ TE monounsaturated fat & $-0 \cdot 193$ & $0 \cdot 014$ & $-0 \cdot 161$ & 0.032 \\
\hline$\%$ TE carbohydrate & & & $0 \cdot 173$ & 0.021 \\
\hline Servings of full-fat dairy & $-0 \cdot 168$ & $0 \cdot 033$ & -0.237 & $0 \cdot 001$ \\
\hline
\end{tabular}

Table 1 presents results for dietary variables that were significantly correlated with maternal anxiety and stress scores. Dietary fat intakes and full-fat dairy showed an inverse association with both states, while AHEI-P score, dietary GI and other food groups rich in dietary fats (e.g. meat, fish, fats \& oils, eggs, desserts \& puddings) did not correlate with either $(\mathrm{P}>0 \cdot 05)$. It was noted that overall fish consumption in this cohort was poor (median servings/day $=0.08$ ). The results were further investigated in linear regression models adjusting for maternal age, ethnicity and pre-pregnancy body mass index. All associations remained significant except for that of trait anxiety with full-fat dairy, and perceived stress with \% TE from carbohydrate.

This study demonstrates a consistent diet-stress interaction in pregnancy such that higher levels of maternal stress and anxiety are associated with lower fat intakes, possibly driven by less dairy consumption. In addition to being an important source of protein and calcium for healthy pregnancy, dairy provides a variety of fatty acids and micronutrients that are involved in maintaining neurological processes linked to maternal mood ${ }^{(5)}$, as well as playing a critical role in fetal neurodevelopment. ${ }^{(6)}$ The potential impact of stress-induced alterations on maternal fat and dairy intakes on pregnancy outcome and fetal development is worthy of further investigation. Prenatal nutrition counselling should also consider maternal psychosocial state as an important factor that may influence dietary behaviours.

1. Dallman MF. (2010) Trends Endocrinol Metab, 21, 159-165.

2. Vaz JdS, Kac G, Emmett P, et al. (2013) PLoS One, 8, e67671.

3. Golding J, Steer C, Emmett P, et al. (2009) Endocrinol, 20, 598-603.

4. Rodriguez-Bernal CL, Rebagliato M, Iniguez C, et al. (2010) Am J Clin Nutr, 91, 1659-1666.

5. Leung BMY \& Kaplan BJ. (2009) J Am Diet Assoc, 109, 1566-1575.

6. Morse NL. (2012) Nutrients, 4, 799-840. 